\title{
Acute renal failure in a patient with epidermolysis bullosa acquisita*
}

\author{
Guowei Zhao ${ }^{1,2}$ \\ Furen Zhang ${ }^{1,3}$
}

\author{
Qing Yang ${ }^{1,3}$
}

DOI: http://dx.doi.org/10.1590/abd1806-4841.20175755

\begin{abstract}
Epidermolysis bullosa acquisita is a severe autoimmune subepidermal bullous disease. In this report, we described for the first time a patient with epidermolysis bullosa acquisita who developed acute renal failure. There is a possibility that epidermolysis bullosa acquisita and acute renal failure's pathogenesis shared some common autoimmune pathways. Moreover, acute blood volume reduction may be another cause of prerenal kidney failure. Further studies are needed to verify our hypothesis.
\end{abstract}

Keywords: Epidermolysis bullosa acquisita; Methylprednisolone; Methylprednisolone hemisuccinate

\section{INTRODUCTION}

Epidermolysis bullosa acquisita (EBA) is a severe autoimmune subepidermal bullous disease, with autoantibodies attached to the cutaneous basement membrane zone (BMZ) of the skin. ${ }^{1}$ EBA was reported to occur along with many systemic diseases, such as systemic lupus erythematosus (SLE), Crohn's disease, Graves' disease, and viral infection, with Crohn's disease being the most frequent. ${ }^{2,3}$ In this report, we described for the first time a patient with EBA who developed acute renal failure (ARF).

\section{CASE REPORT}

A 74-year-old Chinese Han man reported a history of pruritus universalis for six months and bullous eruption on the trunk for one month. He was in good health before the onset of the disease, according to yearly physical examinations. He had no chronic disease and was not regularly taking any medicine. Before being admitted to our hospital, he had been treated with oral prednisone 50 mg for six days, but there was no improvement of the skin lesions. Numerous erosions and blisters were observed on the trunk skin, with a mosaic-like distribution, and the Nikolsky sign was negative (Figure 1). The blood count test and liver and renal function tests were normal three days before the hospital visit.

Skin biopsy revealed a subepidermal blister with lymphocytes and eosinophils infiltrating into the superficial layer of the dermis. Perilesional skin direct immunofluorescence (DIF) revealed linear deposition of IgG, IgA, and C3 at the dermo-epidermal junction. Indirect immunofluorescence (IIF) studies on salt-split skin demonstrated deposits of IgG, IgA. and C3 autoantibodies on the dermal side of the dermo-epidermal separation, pemphigoid circulating antibodies were negative (Figure 2). These findings supported the clinical diagnosis of EBA.

The patient was treated with methylprednisolone at $80 \mathrm{mg}$ per day and ethacridine lactate soaking. After three days of treat-

Work submitted on 03.03.2016

Approved by the Advisory Board and accepted for publication on 27.04.2016

* Work performed at the Shandong Provincial Hospital for Skin Diseases, Shandong University, Shandong, China.

Financial support: None

Conflict of interest: None

Department of Dermatology, Shandong Provincial Hospital for Skin Diseases, Shandong University, Shandong , China.

Department of Dermatology, Central Hospital of ZiBo City, Shandong , China.

Department of Dermatology, Shandong Provincial Institute of Dermatology and Venereology, Shandong Provincial Academy of Medical Science, Shandong, China. 


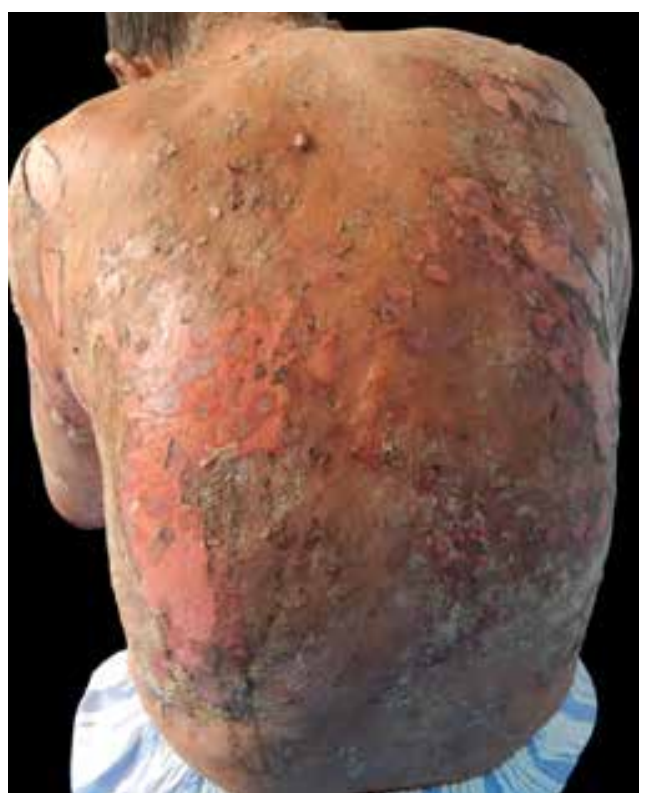

Figure 1: Numerous erosions and blisters on the back

ment, he showed significant improvement: all lesions began to heal with no new vesicle eruptions. However, acute renal failure (ARF) occurred. The liver and renal function tests showed total protein in serum of $56.5 \mathrm{~g} / \mathrm{L}$ (normal is $60-83 \mathrm{~g} / \mathrm{L}$ ), in creatinine of $258.0 \mathrm{umol} / \mathrm{L}$ (the normal is $45-85 \mathrm{umol} / \mathrm{L}$ ), in urea of $19.26 \mathrm{mmol} / \mathrm{L}$ (the normal is $1.7-8.3 \mathrm{mmol} / \mathrm{L}$ ), and in uric acid of $560.0 \mathrm{mmol} / \mathrm{L}$ (the normal is $214-$ $488 \mathrm{mmol} / \mathrm{L}$ ). The blood count test was normal. His urine volume was no more than $400 \mathrm{ml}$ within 24 hours, and routine urinalysis revealed ERY++++, PRO+. The next day, the patient's physical condition became worse: total protein in serum was $45.7 \mathrm{~g} / \mathrm{L}$; creatinine, $384.9 \mathrm{umol} / \mathrm{L}$; urea, $34.67 \mathrm{mmol} / \mathrm{L}$; and uric acid, $748.0 \mathrm{mmol} / \mathrm{L}$.

The patient was transferred to the nephrology department of Qihe county people's hospital for further treatment. He was treated with $0.9 \% \mathrm{NaCl}$ and hydroxyethyl starch (HES) to expand his blood volume, together with intravenous injections of lipid soluble and water soluble vitamins, adenosine triphosphate (ATP), $\mathrm{MgCl}_{2}$, and $\mathrm{KCl}$. Methylprednisolone, at $80 \mathrm{mg}$ per day, was continued. After two weeks of treatment, the patient's creatinine was 105.6 $\mathrm{umol} / \mathrm{L}$ and his urea was $12.23 \mathrm{mmol} / \mathrm{L}$, so the prednisone dose was reduced to $60 \mathrm{mg}$ per day, and no new bullous eruptions occurred. Eight weeks later, his creatinine level was $112.1 \mathrm{umol} / \mathrm{L}$ and urea, $10.23 \mathrm{mmol} / \mathrm{L}$, so the prednisone dose was reduced to $50 \mathrm{mg}$ per day, and very few erythemas were seen.

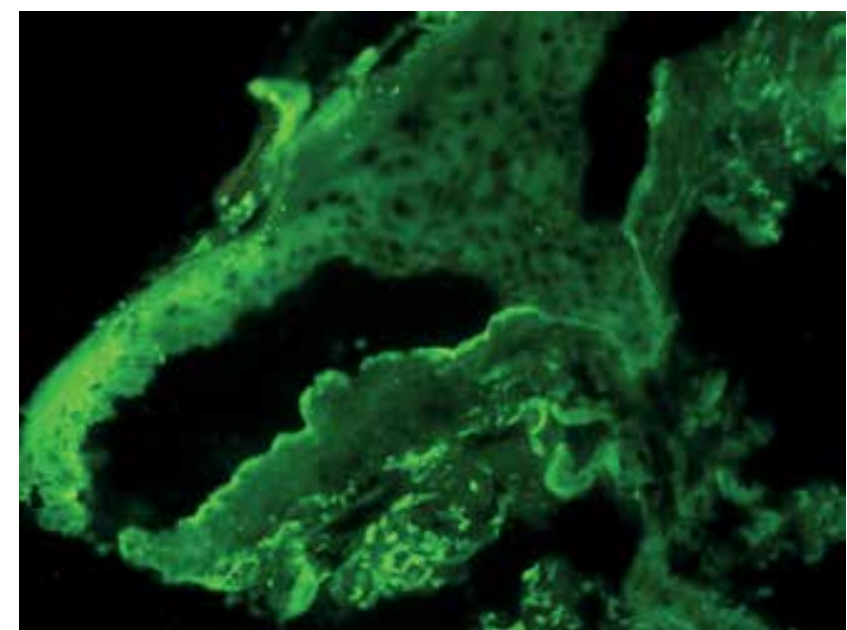

Figure 2: Deposits of IgG, IgA, and C3 autoantibodies on the dermal side of the dermo-epidermal separation (X200)

\section{DISCUSSION}

In this case, ARF occurred shortly after the development of EBA, and rapidly recovered with symptomatic treatment. Although many autoimmune diseases could simultaneously develop in the same patient, it is still unclear why these two diseases occurred almost simultaneously in our patient and whether their pathogeneses are related. It is important to investigate these relationships and provide more effective treatment for EBA in order to better prevent the occurrence of ARF.

EBA is an autoimmune disease, which is associated with a systemic disease in $10-50 \%$ of the patients. ${ }^{4}$ Many different abnormal antibodies and alexins, including IgG, IgA, IgE, IgM, C3, and $\mathrm{C} 4$, have been found in the skin and other tissues of EBA patients. 5-8 Patrick Macken et al. reported a case of crescentic glomerulonephritis in a patient with EBA in 1995, which suggested an autoimmune relationship between EBA and kidney disease. ${ }^{9}$ Therefore, it is possible that EBA and ARF pathogeneses share common autoimmune pathways. In addition, due to the large amount of exudate on the skin lesions, patients with severe EBA suffer from acute blood volume reduction, which may be another cause of pre-renal failure. Further studies, such as renal biopsy, are needed to verify our hypothesis. Nevertheless, many reasons could lead to ARF, such as decreased renal perfusion due to volume depletion, acute heart failure, acute hepatic failure, acute tubular necrosis, obstruction of urinary flow, and prostatic hypertrophy. ${ }^{10,11}$ Urinary tract obstruction and infection could be excluded according to clinical manifestations and laboratory examination. The gold standard of ARF diagnosis is the biopsy, but it was declined by the patient and relatives. Therefore, we could not confirm the exact etiology of ARF in this case. $\square$ 


\section{REFERENCES}

1. Chen M, Kim GH, Prakash L, Woodley DT. Epidermolysis bullosa acquisita: autoimmunity to anchoring fibril collagen. Autoimmunity. 2012;45:91-101.

2. Gupta R, Woodley DT, Chen M. Epidermolysis bullosa acquisita. Clin Dermatol. 2012;30:60-9.

3. Piccolo V, Moscarella E, Errico M, Russo T, Tripodi Cutrì F, Ametrano 0, et al. Could cytomegalovirus infection play a causative role in epidermolysis bullosa acquisita? Eur J Dermatol. 2011;21:607-8.

4. Modiano P, Prost C, Barbaud A, Vignaud JM, Grignon Y, Schmutz JL, et al. Epidermolysis bullosa acquisita and benign schwannoma. J Am Acad Dermatol. 1996:35:472-3.

5. Bauer JW, Schaeppi H, Metze D, Muss W, Pohla-Gubo G, Hametner R, et al. Ocular involvement in IgA-epidermolysis bullosa acquisita. Br J Dermatol. 1999;141:887-92.

6. Miyake H, Morishima Y, Komai R, Hashimoto T, Kishimoto S. Epidermolysis bullosa acquisita: Correlation of IgE levels with disease activity under successful betamethasone/dapsone combination therapy. Acta Derm Venereol. 2001;81:429.

7. Suchniak JM, Diaz LA, Lin MS, Fairley JA. IgM-mediated epidermolysis bullosa acquisita. Arch Dermatol. 2002;138:1385-6.

8. Tokuda Y, Amagai M, Yaoita H, Kawachi S, Ito T, Matsuyama I, et al. A case of an inflammatory variant of epidermolysis bullosa acquisita: chronic bullous dermatosis associated with nonscarring mucosal blisters and circulating $\lg \mathrm{G}$ antitype-VII-collagen antibody. Dermatology. 1998;197:58-61.

9. Macken P, Moorthy AV. Crescentic glomerulonephritis in a patient with epidermolysis bullosa acquisita. Nephron. 1995:70:120.

10. Rahman M, Shad F, Smith MC. Acute kidney injury: a guide to diagnosis and management. Am Fam Physician. 2012 0ct 1;86(7):631-9.

11. Hilton R. Acute renal failure. BMJ. 2006 Oct 14;333:786-90
MAILING ADDRESS:

Furen Zhang

Shandong Provincial Hospital for Skin Diseases, Shandong University,

Jinan,

250000 Shandong, China.

E-mail: zhangfuren@hotmail.com

How to cite this article: Zhao G, Yang Q, Zhang F. Acute renal failure in a patient with epidermolysis bullosa acquisita. An Bras Dermatol. 2017;92(5 Suppl 1): 14-6. 